\title{
On Regular Sets of Polynomials Whose Zeros Lie in Prescribed Domains
}

\author{
M. A. Farag* and M. Nassif*
}

(July 18, 1972)

\begin{abstract}
The relation between the mode of increase of regular basic sets of finite span and the order of magnitude of the zeros of polynomials $\left\{p_{n}(z)\right\}$ belonging to them is investigated. Upper bounds are obtained for the order of the basic sets when the zeros of $p_{n}(z)$ lie either in the unit circle or in a circle whose radius increases in a certain manner with the index $n$ of the polynomial.
\end{abstract}

Key words: Basic sets; Cannon sum of basic sets; lower semiblock matrices; order of basic sets; zeros of polynomials in regular sets.

\section{Introduction}

The relation between the mode of increase of simple sets ${ }^{1}$ and the order of magnitude of the zeros of polynomials belonging to them has been the interest of many authors, of whom we may mention Eweida [1] $]^{2}$ and Nassif [3]. The same problem is studied in the present paper when the sets considered are regular of finite span. To formulate a precise definition of such sets we suppose that $l$ is an integer greater than $1,{ }^{3}$ and the sequence $\left(\mu_{n}\right)$ of integers is constructed so that

$$
\mu_{0}=0,1 \leqslant \mu_{n}-\mu_{n-1} \leqslant l \quad ;(n \geqslant 1) .
$$

Thus, if we put

$$
\nu_{n}=\mu_{n}-\mu_{n-1} \quad ;(n \geqslant 1),
$$

then

$$
1 \leqslant \nu_{n} \leqslant l \quad ; n \leqslant \mu_{n} \leqslant n l .
$$

Let $\left\{p_{n}(z)\right\}$ be a set of polynomials and let $d_{n}$ be the degree of the polynomial $p_{n}(z)$, so that we can write

$$
p_{n}(z)=\sum_{k=0}^{d_{n}} p_{n, k} z^{k}
$$

We shall assume that

$$
d_{0}=0 ; m<d_{m} \leqslant \mu_{n} \quad \text { when } \quad \mu_{n-1}<m<\mu_{n} ; d \mu_{n}=\mu_{n} ;(n \geqslant 1) .
$$

AMS Subject Classification Numbers: Primary 3010.

*An invited paper. Present address, Mathematics Department, Assiut University, Assiut, Egypt.

${ }^{1}$ The reader is supposed to be acquainted with the theory of basic sets of polynomials as given by Whittaker [4,5]. The mode of increase of a basic set is determined by its order and type, c. f. Whittaker [4; pp. 11, 12]. Our concern here is with the order.

${ }^{2}$ Figures in brackets indicate the literature references at the end of this paper.

${ }^{3}$ When $l=1$, the set will be simple. 
When the set $\left\{p_{n}(z)\right\}$ thus defined, is basic it is said to be regular of finite span of bound $l$, and the set is normalized in the sense that

$$
p_{n, d_{n}}=1 \quad ;(n \geqslant 0) .
$$

The matrix $\left(\rho_{n, k}\right)$ of coefficients of such sets is a lower semi-block ${ }^{4}$ matrix whose leading diagonal consists of square matrices $\left(\Delta_{n}\right)$ of the form

$$
\left\{\begin{array}{l}
\Delta_{0}=(1) \\
\Delta_{n}=\left(p_{\left.\mu_{n}-r, \mu_{n}-s\right)}\right) 0 \leqslant r, s \leqslant \nu_{n}-1 ;(n \geqslant 1) .
\end{array}\right.
$$

Let $\delta_{n}$ denote the modulus of the determinant $\left|\Delta_{n}\right| ; \delta_{n}$ should be positive to ensure that the set is basic. Write

$$
\sigma=\liminf _{n \rightarrow \infty} \frac{\log \delta_{1} \delta_{2} \ldots \delta_{n}}{n \log n}
$$

With the above notation, the main results of the present work are formulated in the following theorems. ${ }^{5}$

THEOREM 1: Let $\left\{\mathrm{p}_{\mathrm{n}}(\mathrm{z})\right\}$ be a regular set of polynomials of finite span of bound l, and suppose that the zeros of the polynomials $\left\{\mathrm{p}_{\mathrm{n}}(\mathrm{z})\right\}$ all lie in $|\mathrm{z}| \leqslant 1$. Then the set will be of order not exceeding $\frac{1}{2}(l+1)-\frac{\sigma}{l}$, and this bound is attainable.

THEOREM 2: Let the set $\left\{\mathrm{p}_{\mathrm{n}}(\mathrm{z})\right\}$ be as in Theorem 1 and suppose that the zeros of the polynomial $\mathrm{p}_{\mathrm{n}}(\mathrm{z})$ lie in $|\mathrm{z}| \leqslant \mathrm{n}^{\alpha}$, where $\alpha$ is a positive finite number. Then the order of the set $\left\{\mathrm{p}_{\mathrm{n}}(\mathrm{z})\right\}$ will not exceed $\frac{1}{2}(\alpha+1)(l+1)-\frac{\sigma}{l}$.

\section{Preliminary Results}

We shall establish in this section a lemma, of general type, which is the basis for the proofs of the above theorems. In fact, we shall suppose that the zeros of the polynomial $p_{n}(z)$ lie in $|z| \leqslant \rho_{n}$, where the numbers $\left(\rho_{n}\right)$ accord to the following restrictions.

$$
\rho_{n+1} \geqslant \rho_{n} \geqslant 1 \quad ;(n \geqslant 1),
$$

and there is a finite number $a \geqslant 1$ for which

$$
\left(\rho_{\mu_{n}} / \rho_{\mu_{n-1}}\right)^{\mu_{n-1} \leqslant a^{\nu}} \quad ;(n \geqslant 2) .
$$

In view of (1.4), (1.5), (1.6), and (2.1) it can be verified that

$$
\left|p_{m, k}\right| \leqslant\left(k^{\mu_{n}}\right) \rho_{\mu_{n}}^{\mu_{n}-k} ;\left(0 \leqslant k \leqslant d_{m}, \mu_{n-1}<m \leqslant \mu_{n} ; n \geqslant 1\right)
$$

Inserting (2.3) in (1.7) we find that

$$
\delta_{0}=1 ; \delta_{n}<\lambda_{n} \rho_{\mu_{n}}^{1 / 2 \nu_{n}\left(\nu_{n}-1\right)}=\lambda_{n} T_{n} \rho_{\mu_{n}}^{-\nu_{n}} \quad ;(n \geqslant 1),
$$

${ }^{4}$ c. f. Ibrahim [2; p. 282]

${ }^{5}$ It should be observed that a substitution $z=k_{z^{\prime}}+b$ transforms the circles $|z-b|=k,|z-b|=k n^{\alpha}$ onto the respective circles $\left|z^{\prime}\right|=1,\left|z^{\prime}\right|=n^{\alpha}$ ! Also, according to Whittaker's theorem [4; p. 12] such substitution does not affect the order of the basic set. Hence there is no loss of generality in assuming the zeros to lie in $|z| \leqslant 1$ in Theorem 1 and in $|z| \leqslant n^{\alpha}$ in Theorem 2. We note also that, as far as the order is concerned, the results of Theorems 1 and 2 above reduce to those of Nassif [3; Theorems 6.1,6.2] when $l=1$. 
where

$$
\lambda_{0}=T_{0}=1, \lambda_{n}=\nu_{n} !\left(\begin{array}{c}
\mu_{n} \\
1
\end{array}\right)\left(\begin{array}{c}
\mu_{n} \\
2
\end{array}\right) \ldots\left(\begin{array}{c}
\mu_{n} \\
\nu_{n}-1
\end{array}\right) ; T_{n}=\rho_{\mu_{n}}^{\nu_{n}\left(\nu_{n}+1\right) / 2} ;(n \geqslant 1)
$$

Suppose that $z^{n}$ admits the representation

$$
z^{n}=\sum_{k} \pi_{n, k} p_{k}(z)
$$

The required lemma is concerned with the coefficients $\left(\pi_{n, k}\right)$ :

LEMmA 1: Let the set $\left\{\mathrm{p}_{\mathrm{n}}(\mathrm{z})\right\}$ be as in Theorem 1 and suppose that the zeros of the polynomial $\mathrm{p}_{\mathrm{n}}(\mathrm{z})$ lie in $|\mathrm{z}| \leqslant \rho_{\mathrm{n}}$. Then the coefficients $\left(\pi_{\mathrm{n}, \mathrm{k}}\right)$ satisfy the inequality

$$
\left|\pi_{\mu_{\mathrm{n}}-\mathrm{i}, \mu_{\mathrm{k}}-\mathrm{j}}\right|<\frac{\mathrm{i} !\left(\mu_{\mathrm{n}}-\mathrm{i}\right) ! \lambda_{\mathrm{k}} \lambda_{\mathrm{k}+1} \ldots \lambda_{\mathrm{n}}, \mathrm{T}_{\mathrm{k}} \mathrm{T}_{\mathrm{k}+1} \ldots \mathrm{T}_{\mathrm{n}}}{\rho_{\mu_{\mathrm{n}}}^{\mathrm{i}}\left(\mu_{\mathrm{k}}\right) ! \delta_{\mathrm{k}} \delta_{\mathrm{k}+1} \ldots \delta_{\mathrm{n}}} \mathrm{c}^{\mu_{\mathrm{n}}-\mu_{\mathrm{k}}}
$$

for $0 \leqslant \mathrm{i} \leqslant \nu_{\mathrm{n}}-1 ; 0 \leqslant \mathrm{j} \leqslant \nu_{\mathrm{k}}-1 ; \mathrm{n} \geqslant \mathrm{k} \geqslant 0$, where the constant $\mathrm{c}$ is fixed by

$$
\mathrm{c}=\frac{\mathrm{a}}{\log (1+\mathrm{i} / l)}>\mathrm{a} \geqslant 1
$$

Proof: It should be observed, first of all, that the matrix $\left(\pi_{n, k}\right)$, which, according to (2.6), is the unique inverse of the matrix of coefficients $\left(p_{n, k}\right)$, is of the same structure as this last matrix. Thus, carrying out the product of the matrices $\left(\Delta_{k}\right)$ with their corresponding matrices in $\left(\pi_{n, k}\right)$, we get $\pi_{0,0}=1$, and

$$
\left(p_{\mu_{k-r, \mu_{k}-s}}\right)\left(\pi_{\mu_{k}-t, \mu_{k}-u}\right)=I_{k},\left(0 \leqslant r, s, t, u \leqslant \nu_{k}-1 ; k \geqslant 1\right),
$$

where $I_{k}$ is the unit matrix of order $\nu_{k}$. Applying (2.1), (2.3), (2.4), and (2.5) the following inequality is obtained.

$$
\left|\Pi_{\mu_{k}-i, \mu_{k}-j}\right|<\frac{i !\left(\mu_{k-i}\right) ! \lambda_{k} T_{k}}{\left(\mu_{k}\right) ! \rho_{\mu_{k}}^{i} \delta_{k}} ;\left(0 \leqslant i, j \leqslant \nu_{k}-1 ; k \geqslant 1\right) .
$$

The product is then carried out with respect the remaining elements of the matrices $\left(p_{n, k}\right)$ and $\left(\Pi_{n, k}\right)$. When $k \geqslant 1$, the following equations are formed.

$$
\sum_{s=\mu_{k-1}+1}^{\mu_{n}} p_{\mu_{n}-r, s} \Pi_{s, \mu_{k}-j}=0 ; r=0,1, \ldots, \nu_{n}-1 ;\left(0 \leqslant j \leqslant \nu_{k}-1 ; n \geqslant k \geqslant 1\right) .
$$

These equations can be solved for the coefficients $\left(\Pi_{\mu_{n-i}, \mu_{k-j}}\right) ; i=0,1, \ldots, \nu_{n}-1$, since $\delta_{n}>0$. Appealing to the relations (2.1), (2.3), and (2.5) we obtain

$$
\left|\Pi_{\mu_{n}-i, \mu_{k}-j}\right| \leqslant \frac{\lambda_{n} T_{n}}{\left(\begin{array}{c}
\mu_{n} \\
i
\end{array}\right) \rho_{\mu_{n}}^{i+\nu_{n} \delta_{n}}} \sum_{s=\mu_{k-1}+1}^{\mu_{n-1}}\left(\begin{array}{c}
\mu_{n} \\
s
\end{array}\right) \rho_{\mu_{n}}^{\mu_{n}-s}\left|\Pi_{s, \mu_{k}-j}\right|,
$$

for $0 \leqslant i \leqslant \nu_{n}-1 ; 0 \leqslant j \leqslant \nu_{k}-1 ; n \geqslant k \geqslant 1$. The inequality (2.7) of the lemma will be deduced from (2.11) when $k \geqslant 1$. In fact, it is seen from (2.9) that (2.7) is true for $n=k$. Also, putting $n=k+1$ in (2.11) and applying (1.3), (2.1), (2.2), (2.8), and (2.9) it can be verified that (2.7) is also satisfied for 
$n=k+1$. Moreover, suppose that (2.7) is valid for $n=k, k+1, \ldots, m-1$, then by application of (1.2), (1.3), (2.1), (2.2), (2.4), (2.7), (2.8), and (2.11) and by simple calculation we can arrive at the following relation.

$$
\begin{gathered}
\left|\Pi_{\mu_{m}-i, \mu_{k}-j}\right|<\frac{T_{m} \lambda_{m}}{\left(\begin{array}{c}
\mu_{m} \\
i
\end{array}\right) \rho_{\mu_{m}}^{i+\nu_{m} \delta_{m}}} \sum_{n=k}^{m-1} \sum_{r=0}^{\nu_{n-1}}\left(\begin{array}{c}
\mu_{m} \\
\mu_{n}-r
\end{array}\right) \rho_{\mu_{m}^{m}}^{\mu_{m}-\mu_{n}+r}\left|\Pi_{\mu_{n}-r, \mu_{k}-j}\right| \\
<\frac{i !\left(\mu_{m}-i\right) ! \lambda_{k} \lambda_{k+1} \ldots \lambda_{m} . T_{k} T_{k+1} \ldots T_{m}}{\left(\mu_{k}\right) ! \rho_{\mu_{m}}^{i} \delta_{k} \delta_{k+1} \ldots \delta_{m}} c_{m}^{\mu}{ }_{k}^{-\mu} .
\end{gathered}
$$

Hence the inequality (2.7) of the lemma is true for $n \geqslant k \geqslant 1$.

Now, when $k=0$, (and hence $j=0$ ), the eqs (2.10) assume the form

$$
\sum_{s=0}^{\mu_{n}} P_{\mu_{n}-r, s} \Pi_{s, 0}=0 ;\left(r=0,1, \ldots, \nu_{n}-1 ; n>0\right)
$$

Solving these equations for the coefficients $\left(\Pi_{\mu_{n}-i, 0}\right) ; i=0,1, \ldots \nu_{n}-1$, and proceeding in the same way as before, we easily obtain the inequality

$$
\left|\pi_{\mu_{n}-i, 0}\right|<\frac{i !\left(\mu_{n-i}\right) ! \lambda_{1} \lambda_{2} \ldots \lambda_{n} \cdot T_{1} T_{2} \ldots T_{n}}{\rho_{\mu_{n}}^{i} \delta_{1} \delta_{2} \ldots \delta_{n}} c_{\mu_{n}}
$$

for $0 \leqslant i \leqslant \nu_{n}-1 ; n>0$. Noting that $\lambda_{0}=T_{0}=\lambda_{0}=1 ; \mu_{0}=0$ it will be seen that (2.12) is merely the inequality (2.7) for $k=0$. The lemma is therefore established.

\section{Proof of Theorem 1}

We shall suppose here that the zeros of the polynomials $\left\{p_{n}(z)\right\}$ all lie in $|z| \leqslant 1$. If $M_{n}(r)$ denotes the maximum value of $\left|p_{n}(z)\right|$ in $|z| \leqslant r ; r>0$, then from (1.5) and (1.6) we have

$$
M_{\mu_{k}-j}(r) \leqslant(r+1)^{\mu_{k}} \quad ;\left(0 \leqslant j \leqslant \nu_{k}-1 ; k \geqslant 1\right) .
$$

Moreover, putting ${ }^{6} \rho_{n}=1$ in (2.4), (2.5), and (2.7) we find that

$$
\left\{\begin{array}{l}
\delta_{n}<\lambda_{n}<l \mu{ }_{n}^{\nu_{n}(l-1) / 2}, \\
\left|\pi_{\mu_{n}-i,{ }_{k}{ }^{-j}}\right|<\frac{i !\left(\mu_{n}-i\right) ! \lambda_{k} \lambda_{k+1} \ldots \lambda_{n}}{\left(\mu_{k}\right) ! \delta_{k} \delta_{k+1} \ldots \delta_{n}} c^{\mu_{n}-\mu_{k},} \\
\left(0 \leqslant i \leqslant \nu_{n}-1 ; 0 \leqslant j \leqslant \nu_{k}-1 ; n \geqslant k \geqslant 0\right) ; c=\frac{1}{\log (1+1 / l)} .
\end{array}\right.
$$

Therefore

$$
\lambda_{1} \lambda_{2} \ldots \lambda_{n}<l^{n} \mu_{n}^{\nu_{n}(l-1) / 2} \quad ;(n \geqslant 1)
$$

In the usual notation, the Cannon sum for the set $\left\{p_{n}(z)\right\}$ is

${ }^{6}$ The restrictions (2.1) and (2.2) for the numbers $\left(\rho_{n}\right)$ are satisfied in this case with $a=1$. 


$$
\omega_{n}(r)=\sum_{k}\left|\pi_{n, k}\right| M_{k}(r)
$$

Introduction of (3.1), (3.2), and (3.3) in (3.4) easily leads to the following relation

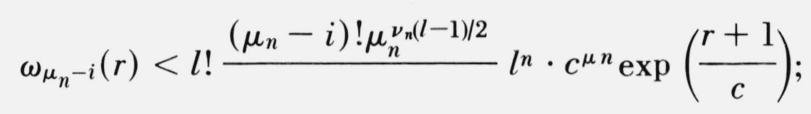

for $0 \leqslant i \leqslant \nu_{n}-1 ; n \geqslant 1$. The order $\omega$ of the basic set $\left\{p_{n}(z)\right\}$ can be evaluated from this relation by application of (1.3) and (1.8), whereby we obtain

$$
\omega \leqslant \frac{1}{2}(l+1)-\frac{\sigma}{l}
$$

as required by Theorem 1 .

\section{Example}

To complete the proof of the theorem a basic set is constructed, according to the conditions of the theorem, such that its order $\omega=\frac{1}{2}(l+1)-\frac{\sigma}{l}$. The following lemma is first proved.

Lemma 2: When $l \geqslant 2$, the function $\mathrm{E}(\mathrm{z})=\sum_{n=0}^{\infty} \mathrm{z}^{\mathrm{n}} l /(\mathrm{n} l)$ ! has at least one zero inside the circle $|\mathbf{z}|=\{(1+1) !\}^{1 / l}$.

Proof: When $l=2, E(z)=\cosh z$, which obviously has the required property. Therefore we shall assume that $l \geqslant 3$ and put

$$
z^{l}=t ; E(z)=e(t) ; f(t)=1+\frac{t}{l !}+\frac{t^{2}}{(2 l) !} ; e(t)=f(t)+r(t)
$$

Now, it is easily seen that when $l \geqslant 3$, the function $f(t)$ has a zero in $-(l+1) !<t<0$, and therefore $f(t)$ has at least one zero in the circle $|t|=(l+1)$ !. Moreover, by actual calculation it can be verified from (4.1) that

$$
|f(t)|>3 / 2 \text { on }|t|=(l+1) ! \quad ;(l \geqslant 3),
$$

and that

$$
\max _{|t|=(l+1) !}|r(t)|<2 / 9 \quad ;(l \leqslant 3) .
$$

Hence, the required result follows by an application of Rouche's theorem.

Now, it is easily seen that the following set $\left\{p_{n}(z)\right\}$ satisfies the conditions of Theorem 1 .

$$
p_{0}(z)=1 ; p_{n l-r}(z)=\left(1+\bar{\epsilon}^{r} z\right)^{n l} ;(0 \leqslant r \leqslant l-1 ; n \geqslant 1),
$$

where $\epsilon=\exp (2 i \pi / l) ; l \geqslant 2$. It is also clear that the zeros of the pr.lynomials all lie on $|z|=1$. From (4.2) we have

$$
p_{n l-r, k}=\left(\begin{array}{c}
n l \\
k
\end{array}\right) \bar{\epsilon}^{r k} ;(0 \leqslant k \leqslant n l ; n \geqslant 1 ; 0 \leqslant r \leqslant l-1) .
$$

Whence, in the notation (2.4) and (2.5), we obtain 


$$
\left\{\begin{array}{l}
\delta_{n}=(l !)^{-1} \lambda_{n} 2^{l(l-1) / 2} \prod_{J=1}^{l-1}|\sin (j \pi / l)|^{j} \\
\lambda_{n}=l !\left(\begin{array}{c}
n l \\
1
\end{array}\right)\left(\begin{array}{c}
n l \\
2
\end{array}\right) \cdots\left(\begin{array}{c}
n l \\
l-1
\end{array}\right) .
\end{array}\right.
$$

Simple calculation based on (4.4) leads to the fact that $\sigma=\frac{1}{2} l(l-1)$. Hence, in view of (3.5), we have to prove that $\omega=1$.

In fact, from the matrix product $\left(p_{n, k}\right)\left(\pi_{n, k}\right)=I$ it follows that

$$
\sum_{k=0}^{n l} p_{n l-r, k} \pi_{k, 0}=0,(r=0,1, \ldots l-1 ; n \geqslant 1) .
$$

Inserting (4.3) in (4.5), adding the results corresponding to $r=0,1, \ldots, l-1$, and putting

$$
b_{k}=\frac{\pi_{k l, 0}}{(k l) !},(k \geqslant 1) ; b_{0}=\pi_{0,0}=1,
$$

we are led to the following relations

$$
\sum_{k=0}^{n} \frac{b_{n-k}}{(k l) !}=0,(n \geqslant 1) .
$$

Writing $E(z)=\sum_{n=0}^{\infty} z^{n l} /(n l)$ ! and $G(z)=\sum_{n=0}^{\infty} b_{n} z^{n l}$, we see that (4.7) implies that $G(z)=1 / E(z)$. Hence, by Lemma 2, we infer that $G(z)$ is regular in $|z|=\rho$, where $\rho<\{(l+1) !\}^{1 / l}$. That is to say

$$
\limsup _{n \rightarrow \infty}\left|b_{n}\right|^{1 / n}=1 / \rho .
$$

Finally, from (4.6) and (4.8) we can deduce that $\omega=1$, and the proof of Theorem 1 is therefore complete.

\section{Proof of Theorem 2}

We now suppose that the zeros of the polynomial $p_{n}(z)$, belonging to the regular set $\left\{p_{n}(z)\right\}$, lie in $|z| \leqslant n^{\alpha}$, where $\alpha$ is a positive number. Hence, in the notation of (3.1), we see that

$$
M_{0}(r)=1, M_{\mu_{k}-j}(r) \leqslant\left\{r+\left(\mu_{k}\right)^{\alpha}\right\} \mu_{k} ;\left(0 \leqslant j \leqslant \nu_{k}-1 ; k \geqslant 1\right) .
$$

Moreover, putting ${ }^{7} \rho_{n}=n^{\alpha}$ in (2.4) and (2.7) we obtain

where

$$
\left\{\begin{array}{l}
\delta_{n}<\lambda_{n} \mu_{n}^{\alpha \nu_{n}\left(\nu_{n}-1\right) / 2} \\
\left|\pi_{\mu_{n}-i, \mu_{k}-j}\right|<\frac{i !\left(\mu_{n-1}\right) ! \lambda_{k} \lambda_{k+1} \ldots \lambda_{n}, U_{k} U_{k+1} \ldots U_{n}}{\left(\mu_{k}\right) ! \delta_{k} \delta_{k+1} \ldots \delta_{n}} c^{\mu_{n}-\mu_{k}} \\
\left(0 \leqslant i \leqslant \nu_{n}-1 ; 0 \leqslant j \leqslant \nu_{k}-1 ; n \geqslant k \geqslant 0\right),
\end{array}\right.
$$

${ }^{7}$ The restrictions (2.1) and (2.2) for the numbers $\left(\rho_{n}\right)$ are still here satisfied with $a=e^{\alpha}>1$. 
so that

$$
c=\frac{e^{\alpha}}{\log (1+i / l)}, U_{k}=\mu_{k}^{\alpha \nu_{k}\left(\nu_{k}+1\right) / 2} \quad ;(k \geqslant 1),
$$

$$
U_{1} U_{2} \ldots U_{n}<\mu_{n}^{\alpha \mu_{n}(l+1) / 2} .
$$

A combination of (3.3), (3.4), (5.1), (5.2), and (5.3) easily yields

$$
\omega_{\mu_{n-i}-i}(r)<l ! \exp \left\{\frac{e^{\alpha}(r+1)}{c}\right\} l_{\mu_{n} c}^{n \alpha l} \mu_{n} \frac{\left(\mu_{n-i}\right) ! \mu{ }_{n}^{\mu_{n}\{\alpha(l+1)+l-1\} / 2}}{\delta_{1} \delta_{2} \ldots \delta_{n}}
$$

for $0 \leqslant i \leqslant \nu_{n}-1 ; n \geqslant 1$. The inequality of Theorem 2 , namely

$$
\omega \leqslant \frac{1}{2}(\alpha+1)(l+1)-\frac{\sigma}{l},
$$

for the order of the basic set $\left\{p_{n}(z)\right\}$, can be easily derived from the relation (5.4). Theorem 2 is therefore established.

\section{References}

[1] Eweida, M. T., Duke Math J. 14, 865-875 (1947).

[2] Ibrahim, S. A., J. of the London Math. Soc. 34, 281-288 (1959).

[3] Nassif, M., Products and zeros of basic sets, Assiut (1958).

[4] Whittaker, J. M., Interpolatory Function Theory (Cambridge Press, 1935).

[5] Whittaker, J. M., Sur les Séries de Base de Polynomes Quelconques, Paris (1949) 